\title{
Óleos essenciais - um breve relato
}

\author{
Essential oils - a brief report
}

\author{
Marise Maleck $^{1,2,3}$, Thiago Dutra Dias ${ }^{1,2}$, Igor Luiz Souza da Cruz ${ }^{1,3}$, Michele Teixeira Serdeiro ${ }^{1,4}$, \\ Nathália Eiras Nascimento ${ }^{1,2}$, Vinicius Marins Carraro ${ }^{1,2}$
}

Como citar esse artigo. Maleck, M; Dias, TD; da Cruz, ILS; Serdeiro, MT; Nascimento, NE; Carraro, VM. Óleos essenciais - um breve relato. Revista Teccen. 2021 Jul./Dez.; 14 (2): 43-49.

\section{Resumo}

As plantas produzem compostos através do seu metabolismo conhecidos como metabólitos primários e metabólitos secundários. Dentre estes encontram-se os óleos essenciais, também conhecidos como óleos voláteis, óleos etéreos ou essência. Nas plantas, desempenham um papel importante na proteção dos vegetais contra bactérias, vírus, fungos, inclusive contra o ataque de herbívoros, além de atrair insetos polinizadores e dispersores de sementes. A síntese e o acúmulo desses óleos nos vegetais geralmente estão associados à presença de estruturas histológicas especializadas, bolsas lisígenas ou esquizolisígenas, células epidérmicas ou tricomas glandulares não se distribuindo de maneira homogênea na planta. A metodologia de extração dos óleos essenciais pode variar de acordo com a localização do óleo na planta e com a sua utilização. Entre os principais métodos pode-se citar a prensagem a frio, arraste por vapor d’água e enfloração. A composição química e a concentração dos óleos voláteis de uma planta são determinadas geneticamente, mas pode sofrer alteração de acordo com os fatores climáticos, e as interações das plantas com outros organismos. Os óleos essenciais apresentam diversas atividades biológicas. Como atividades terapêuticas podem-se citar antimicrobiana, antibacteriana, antifúngica e antiviral, ansiolítica, antidepressiva, anti-inflamatória, antioxidante e anticarcinogênica. Diversos estudos têm demonstrado a eficácia dos óleos essenciais como alternativa no controle de insetos, especialmente mosquitos. Isso se deve, principalmente, porque esses apresentam compostos biodegradáveis e não tóxicos, principalmente para espécies não-alvo, representando uma opção no controle desses vetores.

Palavras-Chave: Metabólito secundário; Insetos vetores; Atividade biológica; Óleos voláteis.

\begin{abstract}
Plants produce compounds through their metabolism known as primary metabolites and secondary metabolites or special metabolites. Among these are essential oils, also known as volatile oils, ethereal oils, or essence. They play an important role not only protecting plants against bacteria, viruses, fungi, the attack of herbivores, but also attracting insect pollinators and seed dispersers. The synthesis and accumulation of these oils in plants are usually associated with the presence of specialized histological structures, lysigenous or schizolysigenous glands, oleiferous channels, epidermal cells or glandular trichomes that are not homogeneously distributed in the plant. The methodology for extracting essential oils can vary according to the location of the oil in the plant and its use. Among the main methods we can mention coldpress extraction, steam distillation and enfleurage. The chemical composition and concentration of volatile oils in a plant are genetically determined but can change according to climatic factors, and plant interactions with other organisms. Essential oils have several biological activities. As therapeutic activities can mention antimicrobial, antibacterial, antifungal, antiviral, anxiolytic, antidepressant, anti-inflammatory, antioxidant and anticarcinogenic. Several studies have shown the effectiveness of essential oils as an alternative to control insects, especially mosquitoes. This is mainly because they have biodegradable and non-toxic compounds, especially for non-target species, representing a option in the control of these vectors.

Keywords: Secondary metabolite; Insect vectors; Biological activity; Volatile oils.
\end{abstract}

Afiliação dos autores:

${ }^{1}$ Laboratório de Insetos Vetores, Universidade de Vassouras, Campi Vassouras e Maricá, RJ, Brasil.

${ }^{2}$ Mestrado Profissional em Ciências Ambientais, Universidade de Vassouras, Vassouras, RJ, Brasil.

${ }^{3}$ Laboratório de Entomologia Médica e Forense, Instituto Oswaldo Cruz, Fiocruz, Rio de Janeiro, RJ, Brasil.

${ }^{4}$ Laboratório de Diptera, Instituto Oswaldo Cruz, Fiocruz, Rio de Janeiro, RJ, Brasil. 


\section{Introdução}

As plantas, sem exceção, produzem compostos através do seu metabolismo que são indispensáveis para garantir sua sobrevivência. Estes compostos podem ser divididos em metabólitos primários, que são encontrados em todas as células vegetais e importantes para o seu desenvolvimento, fundamentais para as funções vitais da planta como nos processos de fotossíntese, respiração, transporte de solutos e assimilação de nutrientes (Souza et al., 2010). E, em metabólitos secundários, sendo através dessas substâncias que as plantas "sentem" e "se fazem sentir" no ambiente, isto é, se comunicam com a sua biota associada. Por essas razões, designadas a esse tipo tão importante de substâncias como "metabólitos especiais” (Gottlieb \& Borin, 2012), que são produzidos como forma de proteção à microrganismos e insetos predadores (Aciole, 2009). Apresentam dentre suas funções: a) alelopatia (supressão do crescimento das plantas vizinhas); b) funções celulares específicas da planta, por exemplo, resistência à salinidade do solo; c) ação tóxica sob agentes patogênicos (bactérias, fungos, vírus, insetos); d) afastamento de animais herbívoros e insetos atração de animais polinizadores para dispersão do pólen da planta (Pichersky \& Gang, 2000; Coelho, 2009). Muitos desses metabólitos são caracterizados por suas propriedades aromáticas (Girard, 2005) e são fontes para o desenvolvimento de produtos que podem ser utilizados no controle de insetos, como os óleos essenciais (Cavalcanti et al., 2004).

Os óleos essenciais (OEs), também conhecidos como óleos voláteis, óleos etéreos ou essências, são misturascomplexasdecompostosnaturaisextremamente voláteis, lipofílicas, geralmente odoríferas e líquidas, com aparência oleosa à temperatura ambiente (Aciole, 2009; Cunha et al., 2012; Heinzmann et al., 2016), e vem sendo amplamente utilizadas na medicina popular, indústria cosmética, farmacêutica e alimentícia (Santos, 2019).

Nas plantas desempenham um papel importante na proteção dos vegetais contra bactérias, vírus, fungos, inclusive contra o ataque de herbívoros, além de atrair insetos polinizadores e dispersores de sementes (Bakkali et al., 2008). Pode-se citar outras funções biológicas que os óleos essenciais exercem sobre as plantas, como a proteção contra o calor e estresse oxidativo, a comunicação entre indivíduos da mesma espécie e o efeito alelopático (Unsicker et al., 2009).

Os OEs são constituídos por componentes em diferentes concentrações, pertencendo a várias classes químicas, entre eles: álcoois simples e terpênicos, éteres ou óxidos, aldeídos, cetonas, ésteres, aminas, amidas, fenóis, heterocíclicos, cumarinas e principalmente os terpenos (Girard, 2005; Heinzmann et al., 2016; Shaalan et al., 2005).
Este estudo se propôs mostrar um breve relato acerca dos óleos essenciais, que vêm crescendo o seu interesse, principalmente, devido as suas atividades bactericida, fungicida e inseticida, e apresentarem menor risco e danos à saúde humana e ao meio ambiente.

\section{A química dos óleos essenciais}

Os óleos essenciais são produzidos e armazenados em estruturas secretoras que podem ser externas ou internas, podendo ser encontradas em várias partes da planta como nas sementes, cascas, caules, raízes, flores, folhas e dos frutos, como por exemplo: sementes (noz moscada), casca (pau-rosa), caule (canela), raízes (gengibre), flores (rosas e jasmim) e frutos (laranja, limão e erva-doce). A sua composição química é muito complexa, um único óleo essencial pode conter 20-200 componentes diferentes (Cunha et al., 2012; Heinzmann et al., 2016).

De acordo com a sua concentração, podem ser chamados de componentes majoritários, que são encontrados em concentrações razoavelmente altas (20 a 95\%), de constituintes secundários, refere-se aos componentes presentes em pequenas quantidades (1 a 20\%), e por último componentes-traço, que é representado por menos de $1 \%$ da mistura. Scherer et al. (2009) analisaram a composição química do óleo essencial do cravo-da-índia (Caryophillus aromaticus Linnaeus, 1753) e três compostos foram considerados majoritários, destacando-se o eugenol, com 83,75\%, seguido pelo $\beta$-cariofileno, com $10,98 \%$, e com $1,26 \%$, o $\alpha$-humuleno, além de outros componentes-traços.

A síntese e o acúmulo desses óleos nos vegetais geralmente estão associados à presença de estruturas histológicas especializadas como células parenquimáticas diferenciadas, bolsas lisígenas ou esquizolisígenas, canais oleíferos, células epidérmicas ou tricomas glandulares não se distribuindo de maneira homogênea na planta (Heinzmann et. al., 2016).

Ainda que todos os órgãos de uma planta possam acumular óleos essenciais, sua composição pode variar de acordo com cada localização. Os óleos essenciais das folhas, caules, rizomas e raízes da espécie vegetal Alpinia galanga (L.) Willd, planta da família do gengibre, foram investigados por Jirovetz et al. (2003). Os óleos essenciais das folhas, caule e rizomas são ricos em 1,8-cineol, apresentando 28,3\%, 31,1\% e $28,4 \%$, respectivamente, enquanto as raízes são ricas em acetato de $\alpha$-fenchila (40,9\%) Olawore et al. (2005) analisaram os óleos essenciais das folhas e frutos de Murraya paniculata (L.) Jack, também conhecida como murta-dos-jardins. No estudo também foi constatado que a composição de ambos os óleos variou qualitativa e quantitativamente. Os principais constituintes do óleo da folha foram $\beta$-ciclocitral (22,9\%), salicilato de 
metila (22,4\%), trans-nerolidol (11,7\%), $\alpha$-cubebeno (7,9\%), (-) - cubenol (6,8\%), $\beta$-cubebeno (5,8\%) e isogermacreno $(5,7 \%)$ e o constituinte mais abundante do óleo essencial da fruta foi o $\beta$ - cariofileno $(43,4 \%)$, seguido de (-) - zingibereno (18,9\%), germacreno $d$ $(8,3 \%), \alpha$-copaeno $(5,5 \%)$ e $\alpha$-humuleno $(5,1 \%)$.

Existem várias metodologias de extração dos óleos essenciais e podem variar de acordo com a localização do óleo na planta e com a sua utilização. Entre os principais métodos pode-se citar a prensagem a frio, arraste por vapor d'água e enfloração (Heinzmann et al., 2016).

A composição dos óleos voláteis de uma planta é determinada geneticamente, mas as condições em que sobrevivem no ambiente são capazes de afetar significativamente a produção de seus metabólitos secundários (Morais, 2009). Fatores climáticos, como temperatura e umidade relativa do ar, a composição do solo em que o vegetal se desenvolve, seu ciclo vegetativo e as interações das plantas com outros organismos são alguns dos aspectos determinantes para a variabilidade química (Masotti et al., 2009).

A composição e a concentração dos componentes dos óleos essenciais de plantas da mesma espécie ou ainda da mesma planta podem variar devido aos aspectos determinantes citados acima. Ribeiro et al. (2018) constataram que a composição química dos óleos essenciais de três espécies do gênero Croton L (Croton blanchetianus Baillon, Croton nepetifolius Baillon e Croton zehntneri Pax et Hoffm) foi influenciada pelo ciclo circadiano e pela sazonalidade. Os autores concluíram que determinados compostos só são produzidos em horários e estações específicas.

Estudo realizado com o óleo da espécie Ocimum gratissimum Linnaeus 1753, popularmente conhecida como alfavacão, demonstrou a variação nos constituintes de acordo com o horário de coleta, tendo sido encontrado $80 \%$ de eugenol na coleta realizada às 12 horas, enquanto as plantas coletadas às 17 horas continham apenas 11\% de eugenol (Craveiro et al., 1981).

A quantidade e a proporção de metabólitos secundários que as plantas produzem é influenciada também pelo seu estágio fenológico. Németh et al. (1993) avaliaram as possíveis mudanças na composição química do óleo essencial de Achillea crithmifolia Waldst. \& Kit 1802 sob diferentes condições ambientais durante suas fases de desenvolvimento. Os autores concluíram que na floração da planta a quantidade da cânfora no óleo essencial diminuiu enquanto que a quantidade de 1,8-cineol aumentou.

\section{Óleos essenciais e atividade biológica}

Desde o início das civilizações, as plantas aromáticas e medicinais vêm mostrando seus benefícios a saúde humana. Os óleos essenciais são, portanto, considerados os primeiros medicamentos utilizados pelo homem primitivo contra enfermidades, devido às suas propriedades químicas e biológicas (Cunha et al., 2012).

Tendo uma composição química bastante complexa, estes metabólitos podem possuir centenas de compostos, e a atividade biológica, normalmente, pode estar relacionada ao sinergismo ou ao seu componente majoritário (Lubbe \& Verpoorte, 2011).

Na natureza, estes metabólitos têm como função de proteger as plantas de pragas ou infecções por conta da sua ação inseticida, antibacteriana e antifúngica. E, a ação antimicrobiana dos óleos essenciais também pode atuar em áreas dos sistemas respiratório, gastrointestinal, nervoso central, circulatório e cardíaco, entre outros distúrbios (Cunha et al., 2012).

As atividades antimicrobiana, especificamente antibacteriana, antifúngica e antiviral, ansiolítica, antidepressiva, anti-inflamatória, antioxidante e anticarcinogênica são as atividades terapêuticas mais bem estudadas.

\section{a)Atividade Antimicrobiana}

O tratamento de infecções microbianas continua sendo, mesmo hoje em dia, um grande obstáculo devido aos efeitos indesejados dos fármacos existentes, mas, principalmente, devido à resistência que microrganismos têm desenvolvido. Assim, urge a necessidade de encontrar alternativas que sejam eficazes e seguras. Vários estudos revelam que óleos essenciais de diversas plantas aromáticas apresentam atividade antimicrobiana, especificamente antibacteriana, antifúngica e antiviral, cuja ação funciona, principalmente, para proteger a planta desses tipos de infecções. Também revelam competência de poder agir em microrganismos que afetam o ser humano.

Há uma gama de espécies que sabidamente tem atividade antimicrobiana como é o caso e do cravo e alecrim (Fu et al., 2007) e cravo da índia e do louro (Cunha \& Roque, 2012), cujas propriedades podem ser vistas na tabela 1 .

\section{b) Atividade sobre o aparelho gastrointestinal}

Na Tabela 2 encontram-se exemplos de óleos essenciais que apresentam atividade gastrointestinal.

Óleos essenciais podem ser utilizados para tratamentos gastrointestinais tanto de caráter simples como uma indisposição gastrointestinal como também auxiliar em situações mais alarmantes como a síndrome do cólon irritado (Dadalioğlu \& Evrendilek, 2004).

\section{c) Atividade sobre o sistema respiratório}

Assim como os óleos que apresentam atividades gastrointestinal, os óleos exemplificados na Tabela 3 
Tabela 1. Óleos essenciais com atividade antimicrobiana.

\begin{tabular}{|c|c|c|}
\hline & Óleo essencial de Loureiro & Óleo essencial de Cravinho \\
\hline Nome Cientifico & Laurus nobilis L., 1753 & Syzygium aromaticum Merr. \& Perry, 1939 \\
\hline Familia & Lauraceae & Myrtaceae \\
\hline Gênero & Laurus & Syzygium \\
\hline Nome Popular & Louro, loureiro-de-apólo, loureiro-dos-poetas & Cravo, cravo-da-india, cravo-de-cabecinha \\
\hline Parte da Planta Utilizada & Folhas & Botões florais secos \\
\hline Composiçäo & $\begin{array}{l}\text { O constituinte principal é o cineol ( } 30-60 \%) \text {, mas } \\
\text { contem aindaacetato de terpenilo (cerca de } \\
10 \%) \text {, sabineno }(7 \%) \text {, metil-eugenol }(5 \%) \text {, pinenos, } \\
\text { 4-terpineol, linalol, p-cimenoe 1,8-cineol }\end{array}$ & Eugenol, acetato de eugenilo e $\beta$-cariofileno \\
\hline Atividade Biológica & $\begin{array}{l}\text { Para além da atividade comprovada contra bactérias } \\
\text { Gram e Gram;, é ainda possivel utilizá-lo como } \\
\text { analgésico em dores musculares, antimicótico e } \\
\text { ainda empsoriases e pediculoses (Cunha et al., } \\
\text { 2012). }\end{array}$ & $\begin{array}{l}\text { O óleo essencial tem ação antibacteriana, antifúngica } \\
\text { e antiviral (FU et al., 2007), sendo utilizado em } \\
\text { inflamaçőes da boca e faringe, cáries dentárias e } \\
\text { otites. Também é associado à estimulação das } \\
\text { secreções gástricas e flatulência devido às grandes } \\
\text { quantidades de eugenol (CUNHA et al., 2012). }\end{array}$ \\
\hline
\end{tabular}

Fonte: Tabela adaptada de Ferreira, 2014.

Tabela 2. Óleos essenciais com atividade gastrointestinal.

\begin{tabular}{|c|c|c|}
\hline & Óleo essencial de Funcho-doce & Óleo essencial de Limoeiro \\
\hline Nome Cientifico & Foeniculum vulgare Mill., 1768 & Citrus limon Burm.fil., 1768 \\
\hline Familia & Apiaceae (Umbelliferae) & Rutaceae \\
\hline Gênero & Foeniculum & Citrus \\
\hline Nome Popular & Erva-doce & Limoeiro-azedo \\
\hline Parte da Planta Utilizada & Frutos e sementes & Pericarpo fresco dos frutos \\
\hline Composiçäo & $\begin{array}{l}\text { Este óleo apresenta um elevado teor de trans-anetol } \\
\text { seguido por componentes como estragol, fenchona, } \\
\text { a-pineno, limoneno, mirceno, canfeno, sabineno, } \beta \text { - } \\
\text { mirceno, } \beta \text {-pineno, a-felandreno, e } \gamma \text {-terpineno }\end{array}$ & $\begin{array}{l}\text { Apresenta como constituinte principal o limoneno } \\
\text { seguido de } \beta \text {-pineno, a-pineno, } \gamma \text {-terpineno, sabineno, } \\
\text { neral, geraniol, acetato de nerilo, acetato de geranilo, } \\
\text { a-terpineno, } \beta \text {-cariofileno, bergamoteno, bisaboleno, } \\
\text { linalol, terpinoleno }\end{array}$ \\
\hline Atividade Biológica & $\begin{array}{l}\text { O óleo essencial apresenta atividade espasmolitica } \\
\text { sobre o músculo liso, mucolitica, digestiva e } \\
\text { antissética (CUNHA et al., 2012). Exibe ainda ação } \\
\text { antibacteriana sobre diversas espécies Gram+e } \\
\text { Gram-, antifúngica e antioxidante (DADALIOGLU E } \\
\text { EVRENDILEK, 2004). }\end{array}$ & $\begin{array}{l}\text { Este óleo essencial confere à planta propriedades } \\
\text { digestivas, mas também } \\
\text { broncoliticas, antimicrobianas e antioxidantes (CUNHA } \\
\text { E ROQUE, 2013). }\end{array}$ \\
\hline
\end{tabular}

Fonte: Tabela adaptada de Ferreira, 2014.

Tabela 3. Óleos essenciais com atividade sobre o sistema respiratório.

\begin{tabular}{|c|c|c|}
\hline & Óleo essencial de Eucalipto & Óleo essencial de Canforeira \\
\hline Nome Cientifico & Eucaliptus globulus Labill., 1800 & Cinnamomum camphora J. Presl, 1825 \\
\hline Familia & Myrtaceae & Lauraceae \\
\hline Gênero & Eucaliptus & Cinnamomum \\
\hline Nome Popular & Eucalipto & Alcanforeira, canforeiro \\
\hline Parte da Planta Utilizada & Folhas secas dos ramos mais antigos & Toda a planta, principalmente a ramagem \\
\hline Composiçäo & $\begin{array}{l}\text { O componente majoritário é o 1,8-cineol, } \\
\text { apresentando em menores quantidades } \alpha \text {-pineno, } \beta \text { - } \\
\text { pineno, canfeno, p-cimeno, limoneno, a-felandreno, } \\
\text { butilaldeido, capronaldeido, aromadendreno e globulol }\end{array}$ & (+) D-cânfora, cineol, a-terpineol, safro \\
\hline Atividade Biológica & $\begin{array}{l}\text { Exerce uma grande ação sobre o sistema } \\
\text { respiratório, graças ao seu componente maioritário. } \\
\text { Este óleo é expectorante, fluidificante de secreções } \\
\text { atuando também como antisséptico (CUNHA et al., } \\
\text { 2012). }\end{array}$ & $\begin{array}{l}\text { Devido ao seu elevado conteúdo em cânfora este } \\
\text { óleo essencial é um estimulante respiratório, } \\
\text { revulsivo, antitússico sendo ainda um bom } \\
\text { antisséptico em doenças catarrais. Pode também ser } \\
\text { usado em fricções para tratamento de mialgias e } \\
\text { situações reumáticas (CUNHA et al., 2012). }\end{array}$ \\
\hline
\end{tabular}

Fonte: Tabela adaptada de Ferreira, 2014. 
também são conhecidos para alívio dos sintomas de patologias respiratórias agudas ou crônicas, de acordo com Cunha, Nogueira e Roque (2012).

\section{Atividade biológica dos óleos essenciais no controle de insetos vetores de doenças}

A utilização de óleos essenciais no controle de insetos tem aumentado por todo o globo, inclusive no Brasil, onde esses estudos têm se desenvolvido amplamente, principalmente por se tratar de um país com uma flora muito rica e diversa. Diversos estudos já evidenciaram a eficácia dos óleos essenciais como alternativa no controle de insetos, especialmente mosquitos. Isso se deve, principalmente, porque esses apresentam compostos biodegradáveis e não tóxicos, principalmente para espécies não-alvo, representando uma opção segura no controle desses vetores (Aciole, 2009).

Diante da imensa gama de trabalhos envolvendo os óleos essenciais extraídos de plantas, pode-se, resumidamente, exemplificar alguns que, destacam sua eficiência e a diversidade de aplicações possíveis em diferentes tipos de organismos, e enfatizando sua importância no estudo do controle de diversas classes de insetos.

A ação do óleo de Lepidium meyenii Walp. (Brassicaceae) contra térmitas (Blattodea: Isoptera), demonstraram que esse fitoproduto é um inibidor de alimentação para estes insetos (Tellez et al., 2002).

Em estudos de atividade larvicida contra Aedes aegypti (Linnaeus, 1762) (Diptera, Culicidae) do óleo essencial de Lippia sidoides Cham. (Verbenaceae), Carvalho et al. (2003) encontraram forte atividade larvicida, pois o óleo causou mortalidade em 100\% das larvas na concentração de $0,017 \%$. Em outro estudo, utilizando o óleo essencial de Cinnamomum osmophloeum Kaneh (Lauraceae), Cheng et al. (2004) avaliaram a composição química e a atividade larvicida contra larvas de Ae. aegypti, onde registraram a presença de monoterpenos, sesquiterpenos e diterpenos na sua constituição química e CL50 oscilando entre 36 e 177 ug/mg, variando de acordo com a composição química analisada.

Ao testar a atividade larvicida do óleo essencial de Zanthoxylum armatum DC (Rutaceae) contra Ae. aegypti, Anopheles stephensi Liston (Diptera: Culicidae) e Culex quinquefasciatus (Say, 1823) (Diptera: Culicidae), Tiwary et al. (2007), determinaram que dentre as espécies estudadas, o $C x$. quinquefasciatus demonstrou maior sensibilidade ao óleo, com $\mathrm{CL}_{50} \mathrm{e}$ $\mathrm{CL}_{95}$ de 49 e 146 ppm, respectivamente, seguido de $A e$. aegypti e An. stephensi, com CL50 na faixa de 54-58 ppm. Estes dados evidenciaram seu possível uso para o controle de formas imaturas dessas espécies.
Em 2008, Costa et al. demonstraram que os fitoprodutos podem ser usados obtendo diferentes respostas a organismos diferentes, ao analisarem a composição química e a atividade antimicrobiana dos óleos essenciais extraídos de folhas de Guatteriopsis spp. (Annonaceae). Nessa pesquisa foram encontradas mono e sesquiterpenos na composição química e atividade antimicrobiana contra fungos (Candida albicans) e bactérias (Bacillus subtilis e Staphylococcus epidermides).

Ao buscar por produtos naturais que possam ser usados como larvicidas contra Ae. aegypti, Gomes et al. (2016) demostraram que o óleo essencial extraído dos rizomas de Zingiber officinale Roscoe (Zingiberaceae), em oito concentrações diferentes (20, 50, 70, 100, 120, 140,150 e $160 \mu \mathrm{g} / \mathrm{mL}$ ) apresenta atividade larvicida. De acordo com esse estudo, a concentração de $20 \mu \mathrm{g} /$ $\mathrm{mL}$ apresentou a menor atividade larvicida, com apenas $10 \%$ de mortalidade, e a concentração de $70 \mu \mathrm{g} / \mathrm{mL}$ e uma mortalidade de $50 \%$. A partir da concentração de $100 \mu \mathrm{g} / \mathrm{mL}$, obteve se a mortalidade de $80 \%$, e a atividade larvicida do óleo essencial começou a crescer exponencialmente a partir dessa concentração, em que $120 \mu \mathrm{g} / \mathrm{mL}, 140 \mu \mathrm{g} / \mathrm{mL}, 150 \mu \mathrm{g} / \mathrm{mL}$, apresentaram $80 \%, 90 \%$ e $90 \%$ de mortalidade, respectivamente, até provocar a morte de $100 \%$ das larvas testadas com a concentração de $160 \mu \mathrm{g} / \mathrm{mL}$.

Albuquerque et al. (2020) avaliaram o potencial larvicida dos óleos essenciais de Piper dilatatum Rich. (Piperaceae) e Piper hostmannianum C. DC. (Piperaceae) para o controle de Ae. aegypti. Nesse estudo concluiu-se que o óleo essencial das folhas de $P$. dilatatum foi mais eficiente em concentrações menores $\left(\mathrm{CL}_{50}=101,96 \mu \mathrm{g} / \mathrm{mL} ; \mathrm{CL}_{90}=189,84 \mu \mathrm{g} / \mathrm{mL}\right)$ contra as larvas de Ae. aegypti, após 24 horas de exposição, quando comparadas ao óleo de $P$. hostmannianum $\left(\mathrm{CL}_{50}=105,01 \mu \mathrm{g} / \mathrm{mL} ; \mathrm{CL}_{90}=262,69 \mu \mathrm{g} / \mathrm{mL}\right)$.

Benelli et al. (2018) demonstraram que óleos essenciais de Pimpinella anisum L. (Apiaceae) e Cuminum cyminum L. (Apiaceae) quando aplicados em outros invertebrados não-alvo, como em adultos e larvas de Harmonia axyridis (Pallas, 1773) (Coleoptera: Coccinellidae) e adultos de Eisenia fetida (Savigny, 1826) (Oligochaeta: Lumbricidae) não apresentou mortalidade ou efeitos adversos expressivos. Essa baixa toxicidade dos óleos essenciais, indica a sua promissora atividade inseticida e, deste modo, o seu uso seguro no meio ambiente.

Em estudos realizados por Lício (2018) com 10 óleos essenciais derivados do eugenol, nomeados por ordem de aplicação de EU2 a EU11, observou -se que no tratamento das larvas de Ae. aegypti na concentração de $100 \mu \mathrm{g} / \mathrm{mL}$ apresentaram mortalidade de $100 \%$ com os óleos denominados EU2, EU3, EU7, EU9 e EU11; acima de $90 \%$ com os derivados EU4 e EU6; acima de 80\% com o óleo EU5 e abaixo $80 \%$ com os derivados 
EU8 e EU10. Quanto ao ciclo de vida, os bioensaios não apresentaram diferença significativa na duração do desenvolvimento do mosquito.

Estes dados corroboram a afirmativa de Albuquerque et al. (2020) quando mencionam que os óleos essenciais, por apresentarem baixa toxicidade, são promissores na estratégia de controle alternativo de vetores, devido a sua eficácia, atuando como larvicidas, inseticidas e interferindo no ciclo de vida dos insetos.

\section{Considerações finais}

Diante dos dados citados nessa revisão quanto as atividades biológicas apresentadas pelos óleos essenciais, pode-se demonstrar a sua interferência sobre os insetos, principalmente pela mortalidade sobre as larvas de Ae. aegypti. Esses estudos confirmaram que os óleos essenciais são ferramentas eficazes no controle da população larval de Ae. aegypti, e consequentemente, capazes de prevenir a transmissão de doenças que possuem o mosquito como vetor, como febre amarela urbana, dengue, Zika e chikungunya.

\section{Agradecimentos}

Os autores agradecem a Fundação de Amparo à Pesquisa do Estado do Rio de Janeiro (FAPERJ) pelo financiamento e suporte ao projeto de pesquisa "Novas estratégias para o controle do mosquito Aedes aegypti, vetor da Dengue, Chikungunya e do vírus Zika: uma abordagem integrada/RedeZIKA\#1) e ao Prof. Dr. José Maria Barbosa-Filho pela revisão do manuscrito.

\section{Referências}

Aciole, S. D. G. (2009). Avaliação da atividade inseticida dos óleos essenciais das plantas amazônicas Annonaceae, Boraginaceae e de Mata Atlântica Myrtaceae como alternativa de controle às larvas de Aedes aegypti (Linnaeus, 1762) (Diptera: Culicidae). Dissertação de Mestrado em Biologia Humana e Ambiente. Universidade de Lisboa.

Albuquerque, J. S., Gama, E., França, L. P., \& Jesus, R. P. (2020). Atividade larvicida dos óleos essenciais de Piper dilatatum e Piper hostmannianum (Piperaceae) para o controle de Aedes aegypti (Culicidae) em laboratório. DêCiência em Foco, 4 (1), 22-28.

Bakkali, F., Averbeck, S., Averbeck, D., \& Idaomar, M. (2008). Biological effects of essential oils-a review. Food and Chemical Toxicology, 46(2), 446-475.

Benelli, G., Pavela, R., Petrelli, R., Cappellacci, L., Canale, A., SenthilNathan, S., \& Maggi, F. (2018). Not just popular spices! Essential oils from Cuminum cyminum and Pimpinella anisum are toxic to insect pests and vectors without affecting non-target invertebrates. Industrial Crops and Products, 124, 236-243.

Carvalho, A. F. U., Melo, V. M. M., Craveiro, A. A., Machado, M. I. L., Batim, M. B., \& Rabelo, E. F. (2003). Larvicidal activity of the essential oil from Lippia sidoides Cham against Aedes aegypti Linn. Memórias do Instituto Oswaldo Cruz, 98(4), 569-571.

Cavalcanti, E. S. B., Morais, S. M. D., Lima, M. A. A., \& Santana, E. W. P. (2004). Larvicidal activity of essencial oils from Brazilian plants against
Aedes aegypti L. Memórias do Instituto Oswaldo Cruz, 99, 541-544.

Cheng, S. S., Liu, J. Y., Tsai, K. H., Chen, W. J., \& Chang, S. T. (2004) Chemical composition and mosquito larvicidal activity of essential oils from leaves of differents Cinnamomum osmophloeum provenances. Journal of Agricultural and Food Chemistry, 52, 4395-4400.

Costa, E. V., Teixeira, S. D., Marques, F. A., Duarte, M. C. T., Delarmelina, C., Pinheiro, M. L. B., Trigo, J. R., \& Maia, B. H. L. N. S. (2008). Chemical composition and antimicrobial activity of the essential oils of the Amazon Guatteriopsis species. Phytochemistry, 69, 1895-1899.

Coelho, M. G. (2009). Óleos essenciais para aromaterapia. Dissertação de Mestrado em Biotecnologia e Bio-empreendedorismo em Plantas Aromáticas e Medicinais, Universidade do Minho.

Craveiro, A. A., Rodrigues, A. S., Andrade, C. H. S., Matos, F. J. A., Alencar, J. W., \& Machado, M. I. L. (1981). Volatile constituents of Brazilian Euphorbiaceae. Genus Croton. Journal of Natural Products, 44(5), 602-608.

Cunha, A. P., Nogueira, M. T., \& Roque, O. R. (2012). Plantas aromáticas e óleos essenciais: composição e aplicações. Fundação Calouste Gulbenkian. Cunha, A. P., \& Roque, O. R. (2013). Aromaterapia - Fundamentos e Utilização, Lisboa, Fundação Calouste Gulbenkian.

Dadalioğlu, I., \& Evrendilek, G. A. (2004). Chemical compositions and antibacterial effects of essential oils of Turkish oregano (Origanum minutiflorum), bay laurel (Laurus nobilis), Spanish lavender (Lavandula stoechas L.), and fennel (Foeniculum vulgare) on common foodborne pathogens. Journal of Agricultural and Food Chemistry, 52(26), 8255-8260.

Ferreira, A. R. A. (2014). Uso de óleos essenciais como agentes terapêuticos. Dissertação de Mestrado em Ciências Farmacêuticas, Universidade Fernando Pessoa.

Fu, Y., Zu, Y., Chen, L., Shi, X., Wang, Z., Sun, S., \& Efferth, T. (2007) Antimicrobial activity of clove and rosemary essential oils alone and in combination. Phytotherapy Research, 21(10), 989-994.

Girard, E. A. (2005). Volume, biomassa e rendimento de óleos essenciais do craveiro (Pimenta pseudocaryophyllus (Gomes) Landrum) em floresta ombrófila mista. Dissertação de Mestrado em Ciências Florestais, Universidade Federal do Paraná.

Gomes, P. R. B., Silva, A. L. S., Pinheiro, H. A., Carvalho, L. L., Lima, H. S., Silva, E. F., Silva, R. P., Louzeiro, C. H., Oliveira, M. B., \& Filho, V. E. M. (2016). Avaliação da atividade larvicida do óleo essencial do Zingiber officinale Roscoe (gengibre) frente ao mosquito Aedes aegypti. Revista Brasileira de Plantas Medicinais, 18, 597-604.

Gottlieb, O. R., \& Borin, M. R. D. M. B. (2012). Químico-biologia quantitativa: um novo paradigma? Química Nova, 35, 2105-2114.

Heinzmann, B. M., Spitzer, V., \& Simões, C. M. O. (2016). Óleos voláteis. In: Simões, C. M. O., Schenkel, E. P., de Mello, J. C. P., Mentz, L. A., \& Petrovick, P. R. Farmacognosia: do produto natural ao medicamento. Artmed Editora.

Jirovetz, L., Buchbauer, G., Shafi, M. P., \& Leela, N. K. (2003). Analysis of the essential oils of the leaves, stems, rhizomes and roots of the medicinal plant Alpinia galanga from southern India. Acta Pharmaceutica-Zagreb -, 53(2), 73-82.

Lício, C. A. P. (2018). Atividade larvicida de óleos essenciais sobre Aedes aegypti. Trabalho de Conclusão de Curso na Graduação em Medicina Veterinária, Universidade de Vassouras.

Lubbe, A., \& Verpoorte, R. (2011). Cultivation of medicinal and aromatic plants for specialty industrial materials. Industrial Crops and Products, 34(1), 785-801.

Masotti, V., Juteau, F., Bessière, J. M., \& Viano, J. (2003). Seasonal and phenological variations of the essential oil from the narrow endemic species Artemisia molinieri and its biological activities. Journal of Agricultural and Food Chemistry, 51(24), 7115-7121.

Morais, L. A. S. (2009). Influência dos fatores abióticos na composição química dos óleos essenciais. Horticultura Brasileira, 27(2), S3299-S3302.

Németh, É., Bernáth, J., \& Héthelyi, É. (1993). Diversity in chemotype reaction affected by ontogenetical and ecological factors. In International Symposium on Medicinal and Aromatic Plants, 344, 178-187. 
Olawore, N. O., Ogunwande, I. A., Ekundayo, O., \& Adeleke, K. A. (2005). Chemical composition of the leaf and fruit essential oils of Murraya paniculata (L.) Jack. (Syn. Murraya exotica Linn.). Flavour and Fragrance Journal, 20(1), 54-56.

Pichersky, E., \& Gang, D. (2000). Genetics and biochemistry of secondary metabolites in plants: an evolutionary perspective. Trends in Plant Science, 5(10), 439-445.

Ribeiro, S. M., Bonilla, O. H., \& Lucena, E. M. P. (2018). Influência da sazonalidade e do ciclo circadiano no rendimento e composição química dos óleos essenciais de Croton spp. da Caatinga. Iheringia, Série Botânica, 73(1), 31-38.

Santos, A. G. S. (2019). Controle de vetores em saúde ambiental: atividade larvicida de óleo essencial de Croton rhamnifolioides encapsulado em nanossistema polimérico frente ao mosquito Aedes aegypti. Dissertação de Mestrado em Gestão Ambiental, Instituto Federal de Educação, Ciência e Tecnologia de Pernambuco.

Shaalan, E. A. S., Canyon, D., Younes, M. W. F., Abdel-Wahab, H., \& Mansour, A. H. (2005). A review of botanical phytochemicals with mosquitocidal potential. Environment International, 31, 1149-1166.

Scherer, R., Wagner, R., Duarte, M. C. T., \& Godoy, H. T. (2009). Composição e atividades antioxidante e antimicrobiana dos óleos essenciais de cravo-daíndia, citronela e palmarosa. Revista Brasileira de Plantas Medicinais, 11, 442-449.

Souza, S., Meira, M., Figueiredo, L., \& Martins, E. (2010). Óleos essenciais: aspectos econômicos e sustentáveis. Enciclopédia Biosfera, 6(10).

Tellez, M. R., Khan, I. A., Kobaisy, M., Schrader, K. K., Dayan, F. E., \& Osbrink, W. (2002). Composition of the essential oil of Lepidium meyenii (Walp.). Phytochemistry, 61(2), 149-155.

Tiwary, M., Naik, S. N., Tewary, D. K., Mittal, P. K., \& Yadav, S. (2007). Chemical composition and larvicidal activities of the essential oil of Zanthoxylum armatum DC (Rutaceae) against three mosquito vectors. Journal of Vector Borne Diseases, 44(3), 198-204.

Unsicker, S. B., Kunert, G., \& Gershenzon, J. (2009). Protective perfumes: the role of vegetative volatiles in plant defense against herbivores. Current Opinion in Plant Biology, 12(4), 479-485. 